Revue musicale OICRM

\title{
Bergsonisme ( Anthologie du PHEM » / Mots clés, 2)
}

\section{Margaux Sladden}

Volume 6, numéro 1, 2019

URI : https://id.erudit.org/iderudit/1062434ar

DOI : https://doi.org/10.7202/1062434ar

Aller au sommaire du numéro

\section{Éditeur(s)}

Observatoire interdisciplinaire de création et recherche en musique (OICRM)

\section{ISSN}

2368-7061 (numérique)

Découvrir la revue

\section{Citer cet article}

Sladden, M. (2019). Bergsonisme (« Anthologie du PHEM » / Mots clés, 2). Revue musicale OICRM, 6(1), 171-196. https://doi.org/10.7202/1062434ar

\section{Résumé de l'article}

Le deuxième chapitre de la section Mots clés de la série "Anthologie du PHEM » entend rendre compte du succès rencontré par la figure de Henri Bergson dans le domaine musical durant la première moitié $\mathrm{du} \mathrm{xx}^{\mathrm{e}}$ siècle. Dans la presse généraliste comme dans les revues musicales spécialisées, à travers l’utilisation de notions forgées initialement par Bergson - telles la « durée » ou l'» intuition » -, un cadre théorique est élaboré, menant à la redéfinition de l'oeuvre musicale et à la défense d'une esthétique moderniste. Les trois articles sélectionnés correspondent à trois moments importants de l'assimilation de la philosophie bergsonienne dans le domaine musical, depuis les premiers jalons conceptuels posés par Louis Laloy en 1914 jusqu’à la récupération des idées phares de Bergson, désormais entrées dans le vocabulaire courant, par le compositeur Désiré Pâque en 1935. 


\title{
Bergsonisme (" Anthologie du PHEM "/ Mots clés, 2)
}

\author{
Margaux Sladden
}

\section{Résumé}

Le deuxième chapitre de la section Mots clés de la série "Anthologie du PHEM » entend rendre compte du succès rencontré par la figure de Henri Bergson dans le domaine musical durant la première moitié $d u x^{e}$ siècle. Dans la presse généraliste comme dans les revues musicales spécialisées, à travers l'utilisation de notions forgées initialement par Bergson - telles la "durée » ou l'« intuition »-, un cadre théorique est élaboré, menant à la redéfinition de l'œuvre musicale et à la défense d'une esthétique moderniste. Les trois articles sélectionnés correspondent à trois moments importants de l'assimilation de la philosophie bergsonienne dans le domaine musical, depuis les premiers jalons conceptuels posés par Louis Laloy en 1914 jusqu'à la récupération des idées phares de Bergson, désormais entrées dans le vocabulaire courant, par le compositeur Désiré Pâque en 1935.

Mots clés : bergsonisme ; esthétique musicale ; modernisme ; philosophie ; presse musicale.

\begin{abstract}
The second chapter of the Keywords section of the series "Anthologie du PHEM" (PHEM source readings) aims to report on the success of Henri Bergson in the musical field during the first half of the 20th century. In the general press as well as in specialized music journals, a theoretical framework based on notions initially forged by Bergson-such as "duration" or "intuition"-is developed, leading to the redefinition of the musical work and the defense of a modernist aesthetic. The three selected articles correspond to crucial moments in the assimilation of Bergsonian philosophy in musical field, from the first conceptual milestones laid down by the music critic Louis Laloy in 1914 to the use of Bergson's key ideas, now part of the current vocabulary, by the composer Désiré Pâque in 1935.
\end{abstract}

Keywords: aesthetics of music; Bergsonism; modernism; musical press; philosophy. 
Les musicographes traitant de questions esthétiques ont accordé un statut privilégié à certains philosophes : ainsi Saint Thomas d'Aquin, Pascal, Schopenhauer, Nietzsche, notamment, sont-ils régulièrement convoqués dans la presse musicale française durant la première moitié $\mathrm{du} \mathrm{xx}^{\mathrm{e}}$ siècle. Parmi les figures retenant l'attention des théoriciens et des critiques, Henri Bergson (1859-1941) joue un rôle particulièrement important. Les raisons de son succès sont en partie d'ordre contextuel : Bergson est actif lors de la période concernée et sa pensée rencontre un vif succès - jusqu'à la veille de la Seconde Guerre mondiale, les champs de la littérature, des arts plastiques, de l'histoire, de la psychologie se voient marqués par une forme de «bergsonisme ambiant» (Combe 2004, Azouvi 2007) ; le domaine musical n'échappe pas à cette imprégnation, d'autant que Bergson lui-même a consacré plusieurs développements importants à la musique et que sa philosophie rend possible, plus fondamentalement, une compréhension renouvelée du phénomène musical. Mise en valeur de l'intuition, confrontée à la raison et au langage, de l'expérience concrète, contre le savoir abstrait et général, de la durée, enfin, essentiellement distincte de l'espace mesurable : la série d'oppositions déployée par le philosophe est largement relayée dans le domaine musical, et les musicographes y ajoutent même un antagonisme supplémentaire, relatif aux différences entre la musique et le langage verbal. Menant dans certains cas à des prises de position extrêmes (Félix Le Norcy promet la substitution de la musique aux mots [Le Norcy 1925], par exemple), une vaste réflexion s'élabore concernant les liens entre poésie et musique et l'irréductibilité radicale de l'œuvre musicale au langage verbal ; nombre d'auteurs traitant de ces questions inscrivent leurs réflexions dans le sillage de Bergson, discutant et reprenant parfois à leur compte ses idées philosophiques.

Les trois articles reproduits ci-dessous ${ }^{1}$ se situent dans un même mouvement de redéfinition de l'œuvre musicale et de sa réception, initié par la lecture de Bergson : il y est plus particulièrement question de l'importance primordiale de la durée et de l'intuition, et des différences entre la musique et la raison. Dans le premier d'entre eux, en 1914, Louis Laloy (grand introducteur du bergsonisme dans la presse musicale aux côtés de Lionel Landry, Charles Koechlin, Boris de Schloezer ou Gabriel Marcel²) se donne pour objectif de familiariser le public avec le " philosophe du temps » et de défendre, en les expliquant pas à pas, certains de ses concepts. De façon sousjacente, la volonté de Laloy est de transposer la philosophie bergsonienne dans le domaine musical ; il signe en cela une étape essentielle de l'assimilation de Bergson. Dans le deuxième article, Charles Koechlin rend compte d'une querelle importante

\footnotetext{
1 Note sur les transcriptions : dans la transcription des textes, nous adaptons tacitement la ponctuation, l'usage des majuscules, des italiques et des guillemets aux normes typographiques modernes. La graphie originale des noms est respectée. Toute autre intervention est signalée en note. Les paragraphes sont numérotés pour faciliter le repérage des passages cités ou glosés dans le commentaire qui précède la transcription des textes. Les coupures sont indiquées par «[...]» ou bien remplacées par un résumé de leur contenu présenté en italique et entre crochets.

2 Voir infra la liste d'articles traitant du bergsonisme durant la première moitié du $\mathrm{xx}^{\mathrm{e}}$ siècle dans les pays francophones.
} 
qui secoue au début des années 1920 le monde intellectuel et musical français en opposant les « bergsoniens » aux « intellectualistes ». Notons que Laloy et Koechlin assument tous deux un caractère programmatique très accentué : les notions bergsoniennes convoquées - respectivement la durée et l'intuition - semblent choisies en ceci qu'elles permettent d'élaborer une certaine conception de l'acte créateur, libéré des carcans traditionnels (comme l'usage d'une pulsation régulière, la construction formelle procédant à des répétitions thématiques ou les enchaînements d'accords stéréotypés) ${ }^{3}$. Le troisième et dernier article va encore plus loin dans ce sens : Désiré Pâque, compositeur, prend la plume en 1935 afin d'expliquer au public son langage musical et il recourt abondamment, pour ce faire, à la philosophie de Bergson. Avec Pâque, nous voyons que la référence bergsonienne permet désormais de se positionner immédiatement comme partisan de l'innovation artistique : reproduisant presque littéralement un cadre théorique mis en place avant lui par des auteurs comme Laloy et Koechlin, le compositeur revendique à son tour son appartenance $\mathrm{au}$ " bergsonisme ", de façon désormais convenue, voire un brin opportuniste, afin de légitimer ses choix stylistiques.

Les trois articles retenus témoignent du large accueil réservé à la philosophie de Bergson dans la presse : ils couvrent une période assez large (de 1914 à 1935) et sont issus de deux organes de presse différents quant à leur format, leur contenu et le lectorat visé : Laloy introduit les idées de Bergson dans Comodia, un journal quotidien, généraliste et centré sur l'actualité culturelle, tandis que les articles de Koechlin et de Pâque sont écrits pour La Revue musicale, qui est probablement la revue la plus favorable à une réflexion nourrie de philosophie durant l'entre-deux-guerres.

\section{«M. Henri Bergson et la musique " par Louis Laloy (Comoedia, 1914)}

\section{COMMENTAIRE}

La philosophie bergsonienne : phénomène de mode et reflet du temps

En 1914, année de son élection à l'Académie française, Henri Bergson est incessamment évoqué dans Comoedia : avant le 6 août, lorsque le début de la guerre interrompt la publication, le nom du philosophe apparaît dans pas moins de 41 numéros différents. Ses concepts fournissent matière à jeux de mots et il est abondamment question de sa nomination à l'Académie et des vives polémiques suscitées par sa philosophie ; sont également mentionnés son public nombreux (et largement féminin) au Collège de France, ainsi qu'un spectacle de revue mettant en

3 Durant la première moitié du siècle, dans le domaine des arts plastiques et de la littérature comme dans le champ musical, la lecture de Bergson sert à défendre le « projet moderniste » (voir notamment à ce sujet Douglass 1983, Burwick et Douglass 1992, Antliff 1993 et 2011, Worms 2003, Milz 2011, Fink 2012). 
scène "Monsieur Bergson ", dont la première chanson est intitulée "Bergson for Ever »... La fréquence avec laquelle Bergson est évoqué indique à quel point sa pensée, à la veille de la Première Guerre mondiale, s'est aussi muée en succès mondain. Afin de montrer que la philosophie bergsonienne ne peut être réduite à un phénomène de mode, Louis Laloy ${ }^{4}$, dans les pages mêmes de Comoedia, va consacrer à son sujet une étude plus substantielle. Selon Laloy, ardent défenseur du bergsonisme, c'est précisément parce que cette philosophie correspond intimement à l'état du monde qu'elle revêt une importance cruciale, à la fois outil pour comprendre l'époque et agent transformateur de celle-ci ; dans son article, le critique entend rendre compte d'un tel processus en ce qui concerne plus particulièrement la création musicale.

Livrant un résumé de la compréhension bergsonienne du temps comme durée, Laloy montre que cette dernière, ne pouvant être ramenée aux mesures spatiales par essence uniformes, a pour seul modèle la conscience humaine, perpétuellement mouvante : "Notre conscience se modifie sans cesse, [...] en chacun de ses instants dure le souvenir ou tout au moins l'influence de l'instant précédent, et ainsi de suite. [...] Ici le passé tout entier se retrouve dans le présent, qui lui-même s'épanouit dans l'avenir » (\$ 8), créant un mouvement continu comparable au déploiement de la vie. Une fois établie la relation entre durée et devenir, Laloy va tâcher d'en montrer les implications musicales et, réciproquement, selon un jeu de résonances à double sens, le rôle particulier que la musique peut endosser dans cette philosophie.

\section{La parenté entre Bergson et Debussy}

Laloy précise la convergence entre les sphères philosophique et musicale en invoquant la figure de Debussy, intimement liée à celle de Bergson ${ }^{5}$. Divers éléments factuels expliquent l'importance de Bergson et de Debussy pour Laloy : celui-ci a été l'élève puis le familier du philosophe, en même temps que l'ami et premier biographe du compositeur (Bergson et Debussy fréquentaient d'ailleurs tous deux le salon tenu par Laloy et ils ont pu s'y rencontrer). Dans l'article dédié au philosophe pour Comoedia, Laloy affirme que « des liens secrets » (\$ 6) unissent Bergson et Debussy : leurs pensées sont non seulement liées, mais elles interagissent l'une avec l'autre (" on peut dire qu'une telle musique ne pouvait se produire que dans le voisinage d'une telle philosophie, et réciproquement », §6). Plus précisément, Laloy montre de façon subtile que l'objet même de la philosophie de Bergson - la critique qu'il livre de l'espace, cantonné à l'abstrait et au général, et la volonté de restreindre son action " pétrifiante » en faveur d'une compréhension des choses guidée par la durée -, cet objet correspond à la forme même que revêtent, chez le philosophe comme chez le compositeur, les développements respectivement conceptuel et musical. Bergson,

4 La fiche biobibliographique sur Louis Laloy est disponible dans la section « Musicographes » du site Presse musicale : http://pressemusicale.oicrm.org/corpus/musicographes/.

$5 \quad$ Le rapprochement entre Debussy et Bergson, déjà esquissé par Paul Landormy quelques années auparavant (Landormy 1910, p. 99), sera ensuite réinvesti durant l'entre-deux-guerres (voir notamment Chennevière 1919, p. 77 et Souvtchinsky 1932, p. 97). 
dans son domaine, élabore un "style philosophique » qui tente de coller au plus près de l'expérience immédiate ; et Laloy souligne à cet égard le fait que la philosophie bergsonienne est loin de constituer un système achevé, clos sur lui-même : il s'agit au contraire d'une pensée en devenir, sujette à modifications et par là éminemment créative. Laloy vante, dans le domaine musical, un processus créatif similaire : "La note viendra s'ajouter à la note [...] par une volonté suivie et sans cesse changeante » $(\$ 11)$ et il précise les caractéristiques formelles auxquelles recourir pour y parvenir : abandon des schémas fixes à répétition pour un développement libre, abandon également d'une métrique régulière et trop fermement marquée ${ }^{6}$. Plusieurs articles précédemment consacrés à Debussy dans diverses revues montrent que, selon Laloy, ces caractéristiques sont précisément celles mises en œuvre par le compositeur (Laloy 1904, p. 106-111 ; Laloy 1905b, p. 244). Et lorsqu'est décrit le libre déploiement de la forme debussyste, modelé sur la vie même et non plus conditionné par des cadres fixes imposés de l'extérieur, c'est bien sûr la philosophie vitaliste de Bergson et plus particulièrement son ouvrage L'évolution créatrice qui servent d'horizon référentiel (Laloy 1910, p. 513-514).

\section{La musique, exemple pour la philosophie}

Laloy, ayant abondamment utilisé Bergson pour rendre compte du langage debussyste, renverse la perspective dans l'article écrit pour Comodia : ici, c'est la philosophie bergsonienne qui est interprétée à la lumière du phénomène musical. "Art du temps », l'œuvre musicale n'est « complète que par son exécution » et en cela inséparable d'une « durée particulière » $(\S 10)$; pour cette raison, son action est particulièrement puissante sur la conscience de l'auditeur (Laloy l'exprime en ces termes : «C'est par cette propriété sans doute que la musique parvient si aisément à nous faire, pendant que nous l'écoutons, une âme qui est la sienne, et non plus la nôtre », §10). En raison de son immatérialité ${ }^{7}$ dénuée de données spatiales (pour autant qu'elle soit " vouée seulement à se chanter soi-même ", § 12), la musique permet dès lors

6 Une intense réflexion gravitant autour de la question du rythme s'élabore durant la première moitié $\mathrm{du} \mathrm{xx}^{\mathrm{e}}$ siècle : l'antagonisme entre rythme libre et mesure, souvent associé d'ailleurs à l'opposition entre temps et espace, entre qualité et quantité, est alors convoqué par un nombre incalculable de critiques et de théoriciens ; à ce sujet, Laloy dénonce pour sa part l'équidistance des temps forts, semblables à « des poteaux télégraphiques le long d'une route départementale » (Laloy 1905a, p. 139).

7 Dans un autre article, Laloy précise son idée : n'étant pas faite « de bois, de métal ou de pierre, la musique bâtit ses édifices avec le sentiment intime de l'existence, et ce qu'elle organise, c'est notre durée » (Laloy 1929, p. 460). Rappelons que le principe d'immatérialité est essentiel chez Bergson : le mode de connaissance qu'il désire instaurer doit donner accès à la vérité dans le domaine de l'immatériel, et sa critique de l'intelligence porte précisément sur le fait que celle-ci se limite à penser la matière - et non la vie, le devenir ou la durée (" l'intelligence et l'instinct [équivalent ici de l'intuition] sont tournés dans deux sens opposés, celle-là vers la matière inerte, celui-ci vers la vie », Bergson [1907]1970, p. 645). Après Laloy, cette conception bergsonienne sera expliquée par Koechlin : "Il semble qu'existent, dans l'humanité pensante, deux tendances contraires, manifestations opposées correspondant aux phénomènes matériels : inertie - mouvement. Immobilité, évolution. Stabilité des anciens principes, théories nouvelles. Caractère définitif, absolu, de la philosophie grecque ; pragmatisme, évolutisme [sic], relativisme, de William James, de Bergson et d'Henri Poincaré » (Koechlin 1922b, p. 117). 
de mener plus loin les recherches entreprises conjointement par les impressionnistes dans le domaine pictural et par les symbolistes en poésie, toujours tributaires pour leur part de la matière, de l'espace et de la raison ; à une époque où l'on clame que « l'espace n'est plus à craindre » (§ 13), il semble que l'œuvre musicale constitue le modèle par excellence pour appréhender, voire compléter, la philosophie ayant initié de tels postulats. Ainsi, la transposition de la pensée bergsonienne dans la sphère musicale montre que les musicographes peuvent endosser un rôle actif à l'égard de cette philosophie, permettant de mener plus loin la réflexion lorsque celle-ci se voit confrontée aux spécificités de la musique - à cet égard, durant l'entre-deux-guerres, Gabriel Marcel et Charles Koechlin proposeront deux études fondatrices, engageant une véritable discussion des thèses bergsoniennes relativement au phénomène musical (Marcel 1925; Koechlin 1926).

\section{TRANSCRIPTION}

[1] Un poète inoffensif a droit aux plus respectueuses condoléances. Il n'en est pas moins vrai que l'Académie française se fût déshonorée en le préférant, de même qu'en préférant qui que ce soit à $\mathrm{M}$. Henri Bergson ${ }^{8}$.

[2] Il est permis d'accepter ou de combattre les considérations de ce philosophe. Nul n'en contestera ni la valeur, ni l'opportunité. Par elles toutes les régions de la connaissance se sont trouvées mises en un nouveau jour. Des difficultés se sont évanouies ; des contradictions se sont expliquées ; des confusions se sont dénoncées ; et il n'est pas un antique problème dont l'étude reprise n'ait fourni des solutions inattendues. Ce fut comme une délivrance, si heureuse d'ailleurs qu'on songeait plus à s'en féliciter qu'à s'en étonner, ou plutôt on ne s'étonnait que d'une chose : de ne pas s'être avisé plus tôt de tout cela. Comme tous les autres arts, l'art de penser n'échappe à la vieillesse que par ces révélations soudaines qui répondent aux questions non formulées et rendent aux notions familières la fraîcheur de la découverte.

[3] La philosophie de M. Bergson a ses adversaires ${ }^{9}$; toutefois on cherche moins à en dissoudre les argumentations qu'à lui faire des procès de tendance. Le rationalisme classique déclare la logique perdue, et la morale en danger, cependant que le gros des savants, cramponnés à leur bloc de matière, défendent qu'on retire à l'atome aucun des attributs, d'ailleurs incompatibles, dont ils ont doté, au hasard de l'expérience, cette abstraction étendue, pesante, invariable et impénétrable ${ }^{10}$. [Le progrès des sciences s'est toujours accompli en dehors des sentiers institutionnels balisés ; exemple de Galilée.]

[4] De tous temps aussi ces persécutions de la personne ou de la doctrine furent vaines, et nous voyons aujourd'hui les idées de $\mathrm{M}$. Bergson à ce point répandues que ceux-là

8 Remportant 19 voix contre 9 accordées au poète spiritualiste Charles de Pomairols, Bergson a été élu à l'Académie française le 12 février 1914 (soit une semaine avant l'article de Laloy).

9 Au sujet des « adversaires » de Bergson, voir l'article de Charles Koechlin ci-dessous.

10 Dans L'évolution créatrice (1907) en particulier, Bergson a critiqué le matérialisme scientifique de la physique, de la chimie et de la biologie, dont il dénonce l'ancrage dans les mathématiques et une conception mécaniste des phénomènes. S'attachant aux opérations de mesure et à l'étude de la matière plutôt qu'à la volonté d'expliquer les processus, ces disciplines ne sont pas à même selon lui de rendre compte de l'évolution de la vie. L'évolution créatrice est accueilli froidement par les scientifiques, notamment par les biologistes auxquels l'ouvrage semblait pourtant spécialement destiné. 
même qui les ignorent ou ne les peuvent saisir en ont cependant quelque pressentiment et se doutent qu'ils penseraient avec ces idées-là, si par accident il leur arrivait de penser. $\mathrm{M}$. Bergson est un homme à la mode, et c'est bien ce que de graves censeurs lui reprochent, comme ils le reprochent aussi à $\mathrm{M}$. Debussy, à $\mathrm{M}$. Rodin ${ }^{11}$, et généralement à tout artiste qui leur refuse le plaisir de le protéger, de le prôner, de le proposer à l'ingratitude du siècle, et de l'admirer tout à leur aise, dans la certitude que la gloire du moins ne lui viendra pas. [5] Il est indiscutable que la gloire ne suit pas toujours la mode, mais il est sans exemple qu'un homme de génie ait été entièrement méconnu par ses contemporains. [Exemples de César Franck et de Beethoven.]

[6] M. Bergson est le philosophe de notre temps, comme M. Debussy en est le musicien, comme M. Rodin en est le sculpteur. Chacun de ces grands esprits manifeste à sa manière l'état de notre civilisation, en même temps qu'il le détermine. C'est pourquoi des liens secrets les unissent, et on peut dire qu'une telle musique ne pouvait se produire que dans le voisinage d'une telle philosophie, et réciproquement.

[7] On sait que M. Bergson prend pour principe de son système, ou plutôt pour foyer commun d'où rayonnent ses conceptions, une analyse très serrée de l'idée de temps ${ }^{12}$.

[8] Nos horloges ne mesurent pas le temps directement. D'ailleurs, nous ne mesurons que les longueurs, et c'est seulement par convention que nous rapportons, par exemple, le degré de chaleur à la dilatation. Nous mesurons le temps par le mouvement, et ce que nous mesurons du mouvement, c'est l'espace parcouru. D'où cette conviction presque invincible que le temps est lui-même une sorte d'espace où l'univers est emporté d'un mouvement uniforme. Il appartenait à $\mathrm{M}$. Bergson de montrer que le seul modèle du temps non déformé par les instruments de mesure nous est fourni par notre conscience. Or notre conscience se modifie sans cesse, mais en chacun de ses instants dure le souvenir ou tout au moins l'influence de l'instant précédent, et ainsi de suite. Elle constitue donc un système entièrement distinct de ceux de la mécanique, où l'on admet que chaque instant ne dépend que de l'instant immédiatement antérieur et peut s'en déduire par le moyen d'une équation différentielle où le temps est pris comme une variable indépendante. Ici le passé tout entier se retrouve dans le présent, qui lui-même s'épanouit dans l'avenir. C'est un développement continu d'une espèce particulière qui échappe aux identités de la mathématique. La personnalité n'est pas, comme on croyait, un milieu indifférent où se projettent l'une après l'autre les perceptions, les sensations, et les réflexions ; elle est formée de ces divers éléments comme la plante est faite de cellules et de vaisseaux. Elle germe, fleurit et meurt ${ }^{13}$.

[9] Par ces observations de psychologie, un monde nouveau nous était ouvert. Ce monde intérieur ne reconnaissait aucune des lois qui règnent sur l'espace, et particulièrement il échappait à la redoutable nécessité que tant de philosophes ont essayé vainement d'éluder et que quelques-uns, en désespoir de cause, et malgré la révolte de notre être, avaient

11 Outre l'effet de mode suscité par ces trois figures, c'est sans doute la volonté de Rodin de capter le «mouvement » dans ses sculptures qui explique le rapprochement effectué avec Bergson, penseur de la vie et de la mobilité. Notons par ailleurs que Rodin fréquentait lui aussi, conjointement à Bergson et à Debussy, le salon tenu par Laloy.

12 C'est dans l'Essai sur les données immédiates de la conscience, en 1889, que Bergson développe pour la première fois ses conceptions relatives au temps et à la durée.

13 La métaphore végétale, récurrente chez Laloy, est également utilisée par Bergson : le philosophe s'est notamment attaché à décrire, dans L'évolution créatrice, la constitution et la vie des végétaux pour étayer ses théories. 
reconnue ${ }^{14}$. Un peu plus tard, élargissant sa théorie, M. Bergson a fait naître l'univers d'un progrès pareil à celui de notre conscience. Par un renversement hardi, il prend pour réalité première le temps, et il faut avouer, en effet, que c'est le temps, non l'espace, dont nous prenons connaissance sans intermédiaire. Partout où l'élan vital n'est pas interrompu, le sentiment paraît et l'action se produit ; aussitôt qu'il s'arrête, notre raison projette dans un espace illusoire une simultanéité impossible. Je ne m'attarderai pas à discuter ce système ; je ne crois pas que son auteur le considère comme achevé, car il reste à expliquer pourquoi l'élan vital est sujet à ces sortes de courts-circuits ${ }^{15}$. Sur plusieurs points, M. Bergson continue et confirme les propositions des philosophes ioniens, qui n'admettaient d'existence que dans le mouvement, donc dans le temps ${ }^{16}$; sur d'autres, il retrouve les spéculations plus profondes des taoïstes chinois, et l'expression même qu'il emploie d'évolution créatrice traduit mot pour mot leur formule usuelle, qui est tsao hoa ${ }^{17}$. Mais les taoïstes poussent plus loin leur critique. M. Bergson démontre avec raison que nous ne pouvons concevoir le néant que par opposition, c'est-à-dire négativement ${ }^{18}$. Il en va exactement de même de l'existence, et c'est de quoi les taoïstes se sont avisés.

[10] Mais il suffit que l'indépendance du temps ait été sauvée quand la science se flattait de l'avoir pour toujours réduit à la servitude de l'espace. La musique est l'art du temps. Sans doute la lecture d'un poème est successive, et même nous ne contemplons une peinture ou une statue qu'en la parcourant des yeux comme un livre. Mais il y a cette différence que la musique n'est complète que par son exécution, et que cette exécution ne dépend pas seulement de la durée qu'il nous plaira de choisir, mais d'une durée particulière, dont la forme et le mouvement sont déterminés. C'est par cette propriété sans doute que la musique parvient si aisément à nous faire, pendant que nous l'écoutons, une âme qui est la sienne, et non plus la nôtre.

[11] Il suit de là que la musique pure est celle qui ne se développe que dans le temps, sans nulle intervention d'espace : celle où la note viendra s'ajouter à la note, et l'accord à l'accord, par une volonté suivie et sans cesse changeante. C'est projeter le temps dans l'espace que de reprendre ce qui fut énoncé déjà ${ }^{19}$, ou de combiner entre eux des éléments

14 Bergson n'est pas le seul, en effet, à lutter contre le rationalisme et le positivisme au tournant du siècle : selon des modalités propres, mais réservant tous une part importante à la mobilité ou au devenir, Schopenhauer, Nietzsche, Emerson et Freud opposent tour à tour à la raison la Volonté, la Volonté de puissance, la Force et l'Inconscient.

15 Avec l'élan vital, Bergson applique sa conception de la durée à l'évolution de la vie et au développement des espèces. Théorisé initialement dans L'évolution créatrice, ce concept sera précisé et complété dans L'énergie spirituelle (1919) et Les deux sources de la morale et de la religion (1932).

16 D'autres que Laloy établiront un parallèle entre la philosophie de Bergson et celle des Ioniens (Héraclite en particulier ; voir notamment Wahl 1943, p. 25). Cependant, affirmant au contraire des philosophes ioniens la persistance de l'existence et la réalité de la substance, Bergson se défendra du rapprochement effectué entre sa pensée et celle d'Héraclite (Bergson [1934]1970, p. 1420).

17 Le parallèle établi entre le bergsonisme et le taoïsme est quant à lui tout à fait original ; Laloy réunit ici deux centres d'intérêt majeurs (en pratiquant couramment la langue, l'auteur bénéficie d'une connaissance approfondie de la civilisation et de la pensée chinoises ; il y consacrera d'ailleurs plusieurs études, traductions, récits fictionnels et autobiographiques et sera chargé d'une mission officielle en Chine en 1931).

18 Bergson développe cette idée dans «L'existence et le néant » (Bergson [1907]1970, p. 737-745).

19 Cette proposition rappelle une affirmation de Bergson : " Si tout est dans le temps, tout change intérieurement, et la même réalité concrète ne se répète jamais. La répétition n'est donc possible que dans l'abstrait : ce qui se répète, c'est tel ou tel aspect que nos sens et surtout notre intelligence ont détaché de la 
prémédités, ou même de diviser le temps en une série d'intervalles égaux. Il ne faut d'ailleurs pas confondre l'œuvre avec le travail qui la façonne : l'artiste n'est nullement obligé de se laisser aller au fil de ses rêveries ; il faut, au contraire, qu'il isole d'abord ce qu'il rapprochera ensuite, et procède à de nombreux essais avant de retrouver la continuité originelle ; car son action ne peut s'exercer que par des moyens empruntés à l'espace. Mais, comme M. Bergson le dit très justement, l'action n'est définie que par son terme, non par les démarches intermédiaires. Dans toute conduite non seulement humaine, mais vivante, la seule politique est celle des résultats ${ }^{20}$.

[12] Dans l'œuvre accomplie, l'espace peut garder un rôle, mais c'est un rôle négatif : il retient, il fixe, il arrête. La création est dans la durée. L'espace lui-même ne prend figure que soulevé par le ferment subtil du temps. Encore faut-il qu'il ne pèse pas trop lourd. L'excès en est funeste, et c'est par protestation contre cet excès que les peintres ont cherché une peinture qui ne dépendît plus des objets, mais des impressions, et les poètes une poésie où les sentiments viendraient sans explication se refléter dans les symboles. Aux musiciens aussi il fallait une musique exempte de préoccupations et vouée seulement à se chanter soi-même. Cette musique nous fut donnée, et c'est elle qui, grâce à l'immatérialité des sons, accomplit les vœux les plus chers du symbolisme et de l'impressionnisme ${ }^{21}$.

[13] Aujourd'hui le péril est conjuré, l'espace n'est plus à craindre. Selon la nature des esprits et les exigences des sujets, on peut faire appel à ses distinctions et à ses égalités, sous condition toutefois de ne faire couler par aucune blessure la sève nourricière du temps. L'artiste en tourment de l'ordre se persuadera que cet ordre doit épouser des contours inconnus et que les œuvres précédentes en donnent l'exemple, non la règle ${ }^{22}$. De même, si la raison revendique ses droits dans la philosophie, il faut que d'avance elle s'engage à mettre sa législation en harmonie avec d'autres coutumes.

réalité, précisément parce que notre action, sur laquelle tout l'effort de notre intelligence est tendu, ne peut se mouvoir que parmi des répétitions. Ainsi, concentrée sur ce qui se répète, uniquement préoccupée de souder le même au même, l'intelligence se détourne de la vision du temps. Elle répugne au fluent et solidifie tout ce qu'elle touche » (Bergson [1907]1970, p. 533-534).

20 Si Bergson écrit en effet que « dans l'action, c'est le résultat qui nous intéresse ; les moyens importent peu pourvu que le but soit atteint » (ibid., p. 747), il envisage cette affirmation de façon nettement critique, ce qui n'est pas relevé par Laloy : c'est par une vision simplifiée et en quelque sorte dégradée que l'esprit, selon Bergson, se transporte directement au but ; au contraire, le philosophe préconise de s'attacher à percevoir le « mouvement s'accomplissant », dans son devenir et sa durée propres.

21 Laloy avait déjà étudié la similarité entre le « debussysme » et les courants symboliste et impressionniste (Laloy 1910, p. 509-511, 515).

22 Émerge dans cette proposition le rapport que Laloy désire instaurer avec la tradition musicale : s'il est essentiel pour un compositeur de se libérer des règles trop contraignantes, cela n'implique pas de rejeter toutes les bonnes choses issues du passé. En réalité, la définition que donne Laloy de la durée bergsonienne ("le passé tout entier se retrouve dans le présent, qui lui-même s'épanouit dans l'avenir ») correspond également à sa conception de l'histoire de la musique - envisagée dans une forme de continuité. Pour cette raison, le modernisme défendu par Laloy, tout comme ce sera le cas chez Koechlin, peut être considéré comme un « modernisme modéré ». 


\section{«D'une vaine dispute - La Musique plaisir de 1'esprit ou jouissance sensuelle " par Charles Koechlin \\ (La Revue musicale, 1921)}

\section{COMMENTAIRE}

\section{La querelle de l'intelligence et de la sensibilité}

Dans son article, Charles Koechlin ${ }^{23}$ prend position sur une querelle intense qui secoue depuis le début des années 1910 le monde philosophique et artistique français. Cette querelle s'est cristallisée autour de la pensée de Bergson, objet à la fois d'un succès fracassant, comme nous l'avons vu, et d'attaques virulentes : le bergsonisme, lui-même critique $\mathrm{du}$ positivisme et $\mathrm{du}$ rationalisme, est pour cette raison perçu par certains (tels Julien Benda, Georges Politzer ou Jacques Maritain) comme une menace contre l'intelligence et la raison. Dans ce contexte, de nombreux penseurs sont poussés à se positionner pour ou contre Bergson, et cela définit deux conceptions de l'œuvre très différentes dans le domaine musical : l'étude nourrie de positivisme scientifique à laquelle est opposée une approche s'appuyant sur 1' "intuition ", la " sensibilité », ou encore l' "inconscient » (bien que ces termes puissent recevoir des acceptions différentes selon qui écrit, l'arrière-fond est presque toujours bergsonien).

À la suite de la reproduction d'un article de Maurice Barrès soulignant l'importance du " plaisir » en musique (Barrès 1920), il semble nécessaire à Koechlin de dresser un bilan de la polémique opposant les « intellectuels » aux " sensitifs ». Il entend résumer, pour ce faire, les positions de chacun des deux camps, mais surtout en dénoncer les idées reçues et les raccourcis de pensée, pour revenir, au-delà des pétitions de principe, aux " choses réelles » de la musique (p. 219). En effet, dès l'entame, Koechlin se montre circonspect vis-à-vis du bienfondé de la querelle, qu'il qualifie de "phrases toutes faites », de « dogmes de chapelle » (p. 219), de « creuse rhétorique » (p. 240, § 10 dans notre transcription). Le critique refuse d'ailleurs de s'enliser dans la polémique : il n'entend pas prendre position («Nous ne voulons point entrer dans le débat », p. 240, $\S 9)$, mais questionner la validité d'un conflit qui structure alors le monde intellectuel français. Koechlin remarque par ailleurs qu'une seconde querelle (gravitant autour de l'œuvre debussyste) vient se greffer sur la polémique suscitée par la philosophie de Bergson : dans le domaine musical, les partisans de l'intellectualisme accusent Debussy et les compositeurs qui s'inscrivent dans sa ligne de viser la seule sensation - or, le critique a déjà fait un sort à une telle conception (Koechlin 1927, p. 91-94).

S'attachant à préciser les rôles respectifs de la sensibilité (comprise dans un premier temps du raisonnement comme plaisir sensitif) et de l'intelligence, à la fois dans les processus de création et de réception de l'œuvre musicale, Koechlin est amené à redéfinir en profondeur ces deux notions. Pour la première, le constat est

23 La fiche biobibliographique sur Charles Koechlin est disponible dans la section « Musicographes » du site Presse musicale: http://pressemusicale.oicrm.org/corpus/musicographes. 
vite dressé : la musique ne se résume pas à un simple plaisir physique ("jamais ce plaisir n'est suffisant », ou encore : " le plaisir n'est pas uniquement physique ; il est musical, esthétique », p. 225). C'est donc à une conception enrichie de la sensibilité qu'appelle Koechlin, et cette conception irrigue tout son article et une grande part de son activité critique (voir notamment Koechlin 1924, 1929a, 1929b et 1929c) ; l'importance accordée à la sensibilité, chez Koechlin, mériterait un développement à part entière, contentons-nous d'en souligner ici le caractère éminemment émotionnel, humain, intime et libre.

Dans un second temps, l'idée d'intelligence se voit-elle aussi réévaluée (p. 226-232 ; 235-238, § 2-7) : comme Laloy avant lui, et suivant ici aussi la philosophie bergsonienne ${ }^{24}$, Koechlin dénonce l'utilisation d'un plan préconçu lors du processus compositionnel, au détriment de la libre inspiration ; à ce titre, le critique vante l'improvisation comme moyen de travail. Glissant du domaine de la création à celui de la réception, il cite ensuite explicitement Bergson pour réviser la conception usuelle de l' « ordre » : l'ordre attendu correspond rarement à l'ordre véritable, et ce dernier, plus souvent présent que perçu, peut revêtir mille formes différentes. De la même façon, la « logique musicale » (parfois nommée " harmonie » ou " harmonieuse logique »), subtile, non généralisable et fidèle au mouvement de la vie, n'est pas comparable à la logique rationnelle. On voit ici la parenté étroite avec Bergson, lequel a consacré une grande part de son effort philosophique à dénoncer le caractère abstrait du langage et de la raison ; dans l'article "Musique et mathématique », Koechlin poursuivra dans la lignée bergsonienne, opposant les qualités aux quantités et l'individualité de l'œuvre musicale aux formules générales (Koechlin 1931, p. 429 et 433). Finalement, tout comme chez Laloy, la musique invite à continuer la réflexion initiée par Bergson, et à appréhender les notions d'intelligence, d'ordre et de logique selon de nouveaux critères.

\section{Le rôle de l'intuition en musique}

Koechlin tâche de montrer que la sensibilité et l'intelligence rationnelle, considérées isolément, constituent des moyens peu satisfaisants pour comprendre la musique ; c'est dès lors au moyen d'une compréhension renouvelée et par la mise en lien de ces deux idées qu'il s'agit d'envisager la signification musicale (le « royaume immense » de la musique, écrit-il, "se situe entre les deux extrêmes », p. 220) : comme Bergson, Koechlin se lance à la recherche d'une synthèse de l'intelligible et du sensible.

Mais on mesure alors la complexité de la tâche ; à ce stade du raisonnement, une fois la querelle désamorcée et tandis qu'il s'agirait de poursuivre l'investigation, les termes employés par Koechlin sont révélateurs des difficultés rencontrées : " art mystérieux et multiple » (p. 220), " extrêmement complexe » (p. 221), " fluide de sensibilité mystérieux » (p. 222), " beauté musicale entière, mystérieuse synthèse, une et multiple, indéfinissable, variant avec les auteurs, avec les œuvres » (p. 224), logique

24 L'influence de Bergson sur Koechlin a déjà fait l'objet d'investigations (Duchesneau 2006, p. 19-26 ; Caron 2010, p. 181-182). 
musicale " mystérieuse et complexe [...], non définissable " (p. 231), " harmonie très diverse qu'on ne saurait définir » (p. 231), " profond mystère » (p. 235, § 1), et ainsi de suite. Finalement, l'idée de "mystérieuse Intuition » (p. 237, § 6) est avancée pour rassembler tout ce qui précède. Notion centrale chez Bergson, car elle constitue le fondement de sa méthode philosophique, l'intuition - présentée avec une majuscule par Koechlin - est mobilisée pour expliquer ce qui conduit, dans le domaine de la création, à la beauté, à la logique, à la sensibilité et à l'intelligence véritables. Mais la démonstration s'arrête là ; cette idée n'est pas développée plus avant : il semble en effet impossible de cerner ce qu'est l'intuition au moyen des mots, outils de la raison $^{25}$, et d'en rendre compte dans un article de nature spéculative. Il est d'ailleurs révélateur qu'à cet endroit précis, Koechlin abandonne momentanément la casquette du théoricien pour prendre la parole en tant que compositeur ("souffrez qu'à titre d'artiste nous respections la sensibilité et l'intuition ", p. 240, § 9); dans le même ordre d'idées, l'auteur ne manque pas de souligner que la musique constitue le milieu par excellence où l'intuition peut se déployer librement ${ }^{26}$.

Nous reproduisons ci-dessous la fin du long article de Koechlin (à partir de la page 235 ; pour plus de facilité, nous débutons la numérotation des paragraphes à 1), conclusion aux développements qui viennent d'être résumés ; l'article dans son intégralité est disponible en ligne et a déjà fait l'objet d'un travail d'annotation par Michel Duchesneau (Koechlin 2006, p. 159-178).

\section{TRANSCRIPTION}

[1] Concluons : peu importe qu'il n'y ait point de symétrie apparente, de thèmes combinés, d'armature visible. Ces éléments ne constituent pas la beauté. Ils ne s'y opposent pas non plus. D'ailleurs ils n'ont rien de particulièrement intellectuel, et leur absence n'est point cause forcée d'impressionisme [sic]. - Alors, on ne voit plus très bien ce que signifie l'opposition première entre impressionistes [sic] et intellectualistes ${ }^{27}$. Elle apparaît comme à côté, en dehors de la musique. Et c'est à peu près (nous n'exagérons guère) comme si l'on prétendait distinguer, chez les mathématiciens, ceux qui savent ou non monter à cheval ; ou bien encore, établir une hiérarchie des receveurs de contributions directes, selon qu'ils sont épris ou non de la Danse. L'infinie diversité de la musique reste loin de cette discussion : elle se développe sur un autre plan que le physique ou l'intellectuel. À notre entendement, le seul sens que puissent avoir parfois ces mots de « musique intellectuelle »,

25 C'est aussi ce qui explique le recours abondant effectué par Koechlin au travail de l'« inconscient » pour rendre compte de l'action de l'intuition : dans cet article, il est tour à tour question d'" instinct inconscient » (p. 232), de " logique créative qui s'ignore elle-même » (p. 236, §5), de " création presque inconsciente » (p. 237, §6) et de « subconscient » (p. 238, § 8).

26 Au sujet des liens particuliers entre intuition et musique, voir aussi Koechlin 1922a, p. 121, note 1; Koechlin 1923, p. 239, note 2 ; Koechlin 1929b, p. 63.

27 Peu friand d'étiquettes, Koechlin s'est à plusieurs reprises élevé contre le terme « impressionnisme ». Que la connotation en soit péjorative (tel musicographe recourant à cette appellation afin de dénoncer l'absence de construction formelle chez un compositeur, par exemple) ou au contraire positive (tel autre louant la sensibilité et la richesse d'évocation de cet artiste), l' "impressionnisme », selon Koechlin, ne correspond à aucune réalité en musique (voir à ce sujet Koechlin 1927, p. 91-94; Koechlin 1934, p. 1-20). 
sera péjoratif et s'appliquera, comme blâme, à des œuvres factices, conçues étroitement, sèchement, loin de la «Déesse adorable». Enfin, pour la vraie logique musicale, qui dépend de l'intuition, du goût, de la beauté qu'on porte en soi, elle est d'ailleurs imprévisible, diverse extrêmement, et ses lois restent cachées dans un profond mystère.

[2] Cette manie de vouloir mettre au rang d'honneur la musique qu'on jugeait intellectuelle, eut quelques résultats fâcheux :

[3] D'abord, de tendre à donner le change sur la valeur de certaines œuvres fabriquées comme industriellement, en série, suivant des recettes et des principes établis ${ }^{28}$.

[4] Ensuite, d'inciter à n'entendre qu'en analysant les formes. D'où une sorte d'obsession, comme chaque fois qu'on juge d'un point de vue spécial, étranger à l'art (exemple : n'apprécier un paysage qu'en raison de « ce qu'il donnera sur la plaque photographique »). Et si l'on persiste à soutenir que ce soit l'intelligence alors qui fonctionne en ces analyses, figeant la réalité, se montrant "incapable de comprendre la vie ", elle ne laisserait pas de mériter les reproches de $\mathrm{M}$. Bergson.

[5] Un autre danger et qui fut sérieux, c'était de mépriser certains moyens de travail. Nous devons ici réhabiliter l'improvisation (même au piano). Chez les maîtres, elle n'est jamais synonyme de désordre. Chez les médiocres, elle les élève quelquefois au-dessus d'eux-mêmes. Certains compositeurs la veulent craindre, ou dédaigner : nous pensons qu'ils se trompent. Il est d'ailleurs tout à fait inexact qu'on se montre moins intelligent et moins « noble », dans la création d'une œuvre d'art, en n'y faisant pas intervenir a priori cette symétrie tenue pour intellectuelle, ou la volonté de suivre un plan défini d'avance. Chacun a le devoir de procéder selon sa nature : nous n'avons à tenir compte que des résultats. Un plan préalable n'est point un facteur essentiel de l'harmonie ; bien mieux, il pourrait y nuire ${ }^{\mathrm{I}}$. Dans tous les cas, il est sage de savoir employer la " raison » où et quand elle est utile ; mais non à tort et à travers. Il n'est pas intelligent d'user de l'intelligence dans les moments où son rôle est de céder la place à d'autres forces. Mais ces forces sont-elles supérieures, - plus belles, plus nobles, ou moins ? Bien avisé qui saurait répondre ; bien outrecuidant qui l'oserait. En art, connaissons-nous l'instinct créateur, d'où il vient, comment il procède ? Mystères. Qu'il y ait à coup sûr une grande part d'intelligence chez l'artiste, soit : ainsi, dans sa vie même, avant la création de l'œuvre ; après, également, lorsqu'il y réfléchit ou qu'il la commente. Berlioz, Rodin furent des penseurs, et c'est parfait. Mais il y a temps pour tout. Combien juste nous apparaît la conception d'un philosophe du XIX ${ }^{e}$ siècle, profond esprit ${ }^{\mathrm{II}}$ : " C'est affaire à la science et à la philosophie

28 Près de dix ans plus tard, en réaction à un article de Lionel Landry, Koechlin reviendra sur la question d'une musique produite en série, de façon industrielle (Landry 1930, p. 33-38 ; Koechlin 1931, p. 424-439).

I [Les notes auxquelles renvoie un chiffre romain figurent dans le texte original de Koechlin] Je sais bien qu'on admire souvent la volonté de Magnard et qu'on le sacre, à cause d'elle, classique. Il faudrait néanmoins s'entendre. La beauté chez lui, ce n'est pas de s'en être tenu au plan traditionnel des symphonies ; c'est de n'avoir rien écrit d'inutile, comprenant le sens véritable des qualités classiques; et surtout, c'est d'avoir mis dans ses œuvres l'expression vivante (non plus théorique ou formelle) de sa volonté. Qui sait d'ailleurs si, plus libre à de certains égards, il n'eût pas été plus entièrement lui-même?

II Charles Dollfus, Révélation et révélateurs, pages 134 à 136 (Michel-Lévy frères, 1858). [Le philosophe et écrivain Charles Dollfus (1827-1913) était l'oncle de Koechlin, et sa pensée religieuse a profondément influencé ce dernier.] 
de satisfaire la raison : l'artiste a qualité pour parler à l'âme, et l'âme veut être émue... Sans doute, une logique parfaite préside à la création des chefs-d'œuvre dans l'art, mais c'est une logique intime qui s'ignore elle-même. Cette ignorance est précieuse et tout artiste doit veiller à ne point la perdre ; qu'il respecte ce mystère intime de son âme où s'accomplit la création : qu'il le garde avec un soin jaloux et se conserve, libre, à l'écart des systèmes. "

[6] Ce qui agit, ce qui crée, c'est la mystérieuse Intuition. Qu'il y ait, dans toute œuvre, un plan, cela n'exige point qu'il ait été conçu d'abord. Et s'il s'est modifié au cours de la réalisation, si même il fut créé presque inconsciemment, au fur et à mesure (l'artiste, maître de son métier, ayant d'instinct le sens de l'ordre musical), le résultat n'en sera pas moins beau, au contraire : tout dépend des cas. Enfin, s'il y a des retouches à faire au sujet des proportions ou des tonalités, c'est la perception juste du beau qui les doit inspirer, et le sens esthétique commande à la composition. Plus tard, si l'on a le loisir et l'envie d'analyser ce qu'on a fait, on le peut : mais tous les vrais musiciens savent bien que ce n'est pas affaire à l'intelligence de déterminer les rapports d'un développement musical, ni l'harmonie d'une modulation.

[7] [Koechlin développe sur l'opposition entre d'une part le sentiment, la sensation, la sensibilité et d'autre part l'intelligence abstraite adepte des " principes a priori ».]

Seule importe la beauté profonde ; et l'on n'y parvient jamais avec de petits raisonnements à côté de l'art véritable. Seule y mène l'émotion, par le secret, l'indéfinissable instinct du beau.

[8] L'instinct ! mot souvent honni... C'est à dessein qu'ici nous l'écrivons. Si l'inspiration, la création musicale restent des mystères non approfondis, il semble que le subconscient (on devrait peut-être dire le surconscient) y joue un rôle primordial : sans qu'un instant, pour cela, l'œuvre cesse d'être celle exactement qui correspond à la personnalité morale et intellectuelle du musicien, tel qu'il est au profond de lui-même. La chose ne surprendra que les esprits superficiels. Ceux des artistes qui cherchent à comprendre comment on trouve et par suite de quels phénomènes l'invention naît du cerveau, ont pu se rendre compte de cet effacement nécessaire de la conscience précise. On a lu dans un livre d'Henri Poincaré un exemple très curieux du travail cérébral inconscient ${ }^{29}$. L'intuition est une puissance cachée et prodigieuse. En mathématiques, que trouverait-on par la simple déduction ? Essayez de résoudre seulement certaines questions de géométrie élémentaire, sans deviner quelle ligne il vous faut tracer ; ou tentez de mettre en équations, rien qu'un simple problème du premier degré : vous aurez parfois à inventer la meilleure "variable". Et je ne parle même pas de tous les merveilleux symboles de l'analyse supérieure... Mais l'intuition, dans la musique, est plus absolue maîtresse encore. Comment, d'une ou deux mesures, conclure par le raisonnement le reste de la phrase commencée et les accords sur quoi nous le bâtirons ? c'est proprement impossible. Seule agit l'invention. Quant à la juger moins noble que le syllogisme et que la Raison pure, cela est outrecuidant plus qu'on ne saurait dire, et vraiment d'une cuistrerie ridicule.

[9] Mais toutes ces belles théories intellectualistes se rattachent à certaine campagne entreprise contre M. H. Bergson. On l'accuse à présent d'avoir calomnié l'intelligence, et peut-être

29 Dans l'article "Comment on invente. Le travail de l'inconscient » et l'ouvrage Science et méthode, Henri Poincaré (1854-1912) a décrit de façon détaillée le travail mental menant à l' «invention mathématique » (Poincaré 1908a et 1908b). Ce travail, constitué d'allers-retours entre l'activité consciente et inconsciente, est par ailleurs basé pour une large part sur la capacité d'intuition, notion centrale de la philosophie de la connaissance forgée par le mathématicien. 
bien ceux qui le prétendent n'ont-ils pas tout à fait compris le sens des phrases de l'illustre philosophe. Après l'avoir porté aux nues (dans le temps où l'on trouvait chez lui des preuves de l'esprit séparé de la matière, l'espoir que l'être humain survivra, la condamnation du déterminisme et celle du " mécanisme » de quelques physiologistes ${ }^{30}$ ), ce ne sont plus aujourd'hui que reproches amers. On blâme sa sympathie pour le Pragmatisme ${ }^{31}$, on ne peut admettre qu'il glorifie le changement sous la forme de l'Évolution créatrice, on en fait un contempteur de la Tradition, de la Raison et de l'Intelligence, on raille qu'il ait exalté la puissance intuitive et qu'il ait rendu à la sensibilité comme à la sensation les places à quoi elles ont droit dans la vie de l'être, - la vie multiple, échappant à l'analyse et qu'on ne peut diviser en compartiments étanches. Nous ne voulons point entrer dans le débat. Mais souffrez qu'à titre d'artiste nous respections la sensibilité et l'intuition. "Le plaisir de sentir est-il donc si fort ? » se demande Bossuet ${ }^{\mathrm{III}}$ en l'épigraphe d'un livre de M. Benda

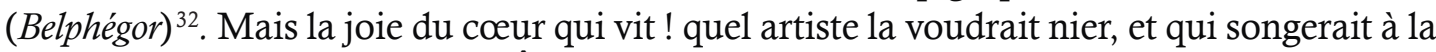
placer au-dessous de la raison ? Ô l'orgueil extrême de prétendre rabaisser la puissance qui dicte l'inspiration, instinct mystérieux et sublime! D'où vient que des hommes se refusent à entendre que si leur être profond ${ }^{\mathrm{IV}}$ crée une œuvre, cette création fournit peut-être la meilleure preuve d'un élément divin dans le monde ${ }^{33}$ ?

[10] Mais résumons. Il nous a semblé absurde d'opposer l'illusoire intellectualisme de la musique dite classique, et le prétendu impressionisme [sic] (esclave de la sensation) qu'on reproche à la moderne. Celle-ci est construite. Celle-là ne laisse pas d'être sensible; et rappelons qu'à l'occasion elle n'ignore point le charme ni les timbres rares ${ }^{\mathrm{V}}$. Ce contraste donc, je n'y vois qu'artifice et creuse rhétorique. Le fait véritable, c'est que l'œuvre d'art est un tout indissoluble ${ }^{\mathrm{VI}}$ où se mêlent et réagissent mutuellement, sensations, associations d'idées, émotions, harmonies de la forme. L'amour de ce qu'on veut chercher d'intellectuel dans la musique serait particulièrement dangereux à l'heure actuelle, en raison de

30 C'est dans Matière et mémoire (1896) que Bergson questionne le rapport entre l'esprit et la matière ; la critique du déterminisme et du mécanisme, annoncée dès l'Essai sur les données immédiates de la conscience (1889), traversera quant à elle toute la philosophie de Bergson.

31 Bergsonisme et pragmatisme cherchent l'un et l'autre à construire une philosophie au plus près du concret et du particulier, en opposition aux abstractions et à l'intellectualisme. Des liens profonds unissent d'ailleurs Bergson et William James (1842-1910), l'un des fondateurs du pragmatisme : James est l'un des premiers à avoir attiré l'attention du public anglais et américain sur la philosophie de Bergson et il a participé à la traduction anglaise de L'évolution créatrice ; Bergson, quant à lui, a rédigé en 1911 la préface à la version française de l'ouvrage Le pragmatisme de James, qui sera reprise dans La pensée et le mouvant (Bergson [1934]1970)

III Bossuet est un ingrat. Sans la sensibilité, que serait donc la religion chrétienne ? Et la Raison humaine ne passa-t-elle point longtemps pour une orgueilleuse adversaire des dogmes?

32 Dans Belphégor. Essai sur l'esthétique de la présente société française, Julien Benda dénonce la recherche, par le public français de son époque, des émotions et sensations suscitées par l'œuvre d'art au détriment du plaisir intellectuel et rationnel ; il y livre également une critique acerbe du bergsonisme (Benda 1918). Un an après l'article de Koechlin, Paul Landormy réalisera un compte rendu critique de l'ouvrage de Benda (Landormy 1922).

IV «Tu portes en toi un ami sublime » (Baghavad-Gitâ).

33 À propos de la conception du religieux chez Koechlin, voir Caron 2010, p. 175-184.

V Témoin ce merveilleux air de basse de la Passion selon Saint Jean, avec accompagnement de luth et violes d'amour.

VI Et le phénomène de l'art apparaît alors bien différent de ce qu'en disent les philosophes obéissant aux habitudes d'esprit du XVII ${ }^{\mathrm{e}}$ siècle. 
ce mouvement d'intellectualisme hors de propos, qui risquerait dans notre art d'écarter les jeunes musiciens de la sensibilité, comme de l'amour d'une belle matière musicale. [Koechlin dénonce le caractère mécanique et simpliste de la " musique à l'emporte-pièce » et des musiques populaires. Il vante au contraire le raffinement pur, simple, grec.]

[11] [...]

[12] Laissons les musiciens aller vers la beauté, sans leur chercher noise de trop, ou de trop peu de symétrie ; sans déplorer l'absence ou la présence de certaines formes. L'artiste ne réalise quelque chose de la beauté qu'il sent en soi, qu'à la condition d'être libre. Nous avons tenté de soutenir que cette indépendance est logique et traditionnelle ${ }^{34}$. Quant aux œuvres, que le public les veuille bien juger de bonne foi, sans les clichés des manuels, les expertises des critiques, les repères des analyses. Il n'est point d'aide plus fallacieuse. N'observez pas la beauté des étoiles doubles ou des nébuleuses à travers les fils du réticule : votre télescope n'en a que faire. Et si, malgré tout, ces fils vous semblaient utiles, n'oubliez pas cependant, n'oubliez jamais que "c'est l'opticien qui les y a mis ${ }^{\mathrm{VII}}$ ».

\section{" Réponse à quelques objections " par Désiré Pâque (La Revue musicale, 1935)}

\section{COMMENTAIRE}

\section{Du positivisme au bergsonisme}

La « Réponse à quelques objections » de Désiré Pâque ${ }^{35}$ est 1'avant-dernier d'une série de huit articles parus entre 1930 et 1936 dans La Revue musicale. Le compositeur, peu connu du public, y expose ses conceptions esthétiques et revendique son importance historique, notamment en ce qui concerne l'invention de l'atonalité (Pâque 1930b, p. 138-139). Ces articles arrivent près de 40 ans après les premières manifestations des choix esthétiques dont ils entendent rendre compte : c'est en 1892 , dans les Vingt leçons de lecture musicale, op. 21, que Pâque tournait pour la première fois le dos à une tonalité définie, et en 1895 qu'il composait une sonate pour trio à clavier "sans tonalité fixe et préétablie ». Mais les années 1930, dernière décennie de la vie du compositeur, correspondent à une période créative prolifique (il écrit près d'un quart de son œuvre entre 1927 et 1939) ainsi qu'à un tournant au point de vue de ses

\footnotetext{
34 Voir note 22 : Koechlin rejoint ici Laloy en ce qui concerne la mise en valeur d'une certaine forme de tradition.

VII Anatole France. [Anatole France, dans son roman Les Dieux ont soif, met en scène l'opticien Fortuné Trubert, mais rien ne permet d'affirmer avec certitude que c'est à ce personnage que fait allusion Koechlin.]

35 Compositeur belge largement méconnu, Désiré Pâque (1867-1939) a pourtant élaboré dès le début des années 1890 un langage musical extrêmement novateur, tournant le dos dans certaines œuvres à la tonalité, à la métrique régulière et à l'approche traditionnelle du développement. À partir des années 1920, il rend compte de sa démarche esthétique dans plusieurs articles rédigés entre autres pour Le Guide du Concert, Le Guide musical ou La Revue musicale.
} 
conceptions esthétiques : jusque-là sous l'influence prédominante de Charles Lalo ${ }^{36}$, dont l'Esquisse d'une esthétique musicale scientifique (Lalo 1908) et son ancrage dans une méthode positiviste et rationaliste l'avaient fortement marqué, Pâque traverse au début des années 1930 une crise de confiance dans la capacité de l'investigation scientifique à dévoiler la signification de la musique. Ce revirement intellectuel apparaît pour la première fois de façon nette dans un article de 1932 au titre révélateur : "Le mystère dans l'art » (Pâque 1932). Prenant délibérément le contrepied d'une proposition de Lalo, Pâque y affirme d'entrée de jeu : "Vraiment tout cet appareil scientifique et matériel n'explique pas le tout de l'art, il y a du "mystère et de l'inconnaissable" " (p. 362), et il critique les approches de l'œuvre musicale fondées sur les mathématiques et l'acoustique, dont il dénonce la " positivité rigide ", le " cadre étroit, vicieux et étouffant ", le " matérialisme anémique » (p. 364). Sur la base de tels postulats, il n'est guère étonnant que Pâque privilégie désormais la référence bergsonienne, très différente de la perspective adoptée par Lalo (lequel livrera d'ailleurs une critique du bergsonisme quelques années plus tard [Lalo 1941]). Bergson est convoqué une première fois sous la plume de Pâque en 1933 (Pâque 1933, p. 108) et à nouveau en 1935, dans la "Réponse à quelques objections ». On retrouve dans ce deuxième article un patchwork de références : L'évolution créatrice de Bergson au premier chef, mais aussi, en bonne place, la pensée d'Édouard Le Roy ; en réalité, ces deux philosophes partagent un horizon conceptuel commun ${ }^{37}$. Dans son article, Pâque reproduit une série de raisonnements déjà établis avant lui par Laloy et Koechlin : critique des abstractions générées par le travail de l'intelligence et par la suprématie de la méthode scientifique (Pâque, pour sa part, mentionne le "fanatisme scientifico-matérialiste », $\S 2$ ) ; cette critique aboutit, tout comme chez Laloy et Koechlin, à la remise en question d'un cadre formel prédéfini et plus particulièrement de l'usage de la répétition thématique littérale. En 1933, Pâque écrivait déjà :

Il faut dégager les données immédiates de la conscience musicale ${ }^{38}$ de toutes les superstructures, idées associées, habitudes acquises, conventions ou préjugés inaperçus qui les dissimulent ou les déforment ; ainsi, telle création qui est comme une genèse perpétuelle, comme une continuité de jaillissement, comme une immense efflorescence d'imprévisible nouveauté, deviendra la conception musicale (ibid., p. 108).

On voit la proximité d'une telle proposition avec la conception, déjà formulée par Laloy et défendue à sa façon par Koechlin, selon laquelle la création musicale doit épouser le concept bergsonien de durée. Une pensée analogue s'y trouve déployée : est tout d'abord posé le principe d'immatérialité de l'œuvre musicale-la musique, « art par

36 Président de la Société française d'esthétique et l'un des directeurs de la Revue d'esthétique, Charles Lalo (1877-1953) conçoit l'esthétique comme une discipline scientifique, objectivable, qu'il base sur l'observation mathématique, psychologique, physiologique et sociologique.

37 Voir note I ci-dessous.

38 Relevons au passage l'usage d'une expression typiquement bergsonienne, transposée ici dans le domaine musical. 
excellence grâce à sa quasi-immatérialité " (Pâque 1933, p. 109³9) est " mouvement, énergie " (Pâque 1936, p. 294) ; Pâque est ensuite amené à souligner le profond mystère auquel elle nous confronte et, enfin, à reconnaître l'intuition comme seule voie d'accès. Pas plus que chez Koechlin, l'intuition n'est ici définie ; elle dépasse simplement, selon Pâque, la conscience rationnelle et le compositeur évoque, pour sa part, "le sens intuitif, ce prolongement du sub-conscient " (Pâque 1932, p. 364). Encore une fois, un projet sous-tend l'ensemble de ces développements et si Bergson est mobilisé, c'est avant tout pour légitimer l'innovation dans le domaine artistique. Étant donné que ses propos techniques sont sans conteste plus intéressants que ses revendications théoriques (lesquelles regorgent d'affirmations hâtives), il reste encore à étudier la façon dont un tel projet se concrétise, au point de vue musical, chez le compositeur.

\section{Manifestation en musique}

Le cœur de l'esthétique de Désiré Pâque réside dans le principe d'« adjonction constante $^{40} »$ : déterminant l'aspect mélodique, élément premier de la musique, l'adjonction constante se substitue au développement thématique traditionnel (dont les compositeurs ont largement abusé, selon Pâque) et constitue une réaction à l'encontre de l' « intellectualisme » en musique. Concrètement, cette technique consiste pour le compositeur à abandonner toute forme fixe au profit d'une plus grande part réservée à l'" inspiration ": en découle la multiplication des motifs - lesquels, s'ils donnent tout de même lieu à une répétition, ne doivent en aucun cas être dénaturés par le morcellement ; une mélodie, explique Pâque, est une « ligne sinueuse [...] faite de continuité " et " toute brisure lui nuit " (Pâque 1930a, p. 123). Mais l'idéal visé est la disparition pure et simple de toute notion de thème afin de produire une musique en perpétuel devenir, sans aucun retour en arrière ; Pâque vante à ce titre l'idée de "haute improvisation ", telle qu'on la rencontrait déjà chez Koechlin : «Il ne doit plus se produire d'entraves au libre - mais ordonné-développement de l'art, par des règles arbitraires et abusives qui ne reposent généralement que sur la crainte du non-connu, de l'imprévu " (Pâque 1930b, p. 139). Un tel programme artistique rappelle l'élan vital bergsonien, lequel génère de façon incessante et dynamique des formes imprévisibles ; mouvement s'imprimant dans la durée qui explique l'évolution du vivant et la liberté de cette évolution, l'élan vital est théorisé par Bergson dans l'ouvrage L'évolution créatrice - et c'est par ces deux termes, précisément, que Pâque achève son article de 1935 (§ 15).

Deux conséquences techniques découlent de tout ce qui précède : abandon de la barre de mesure pour rapprocher le rythme de la " vie » (ibid., p. 140 ; Pâque 1934, p.79-80), selon un mouvement d'émancipation qui a déjà été repéré dans les propos

40 L'adjonction constante est principalement théorisée dans Pâque 1930a, p. 120-127. 
de Laloy, et rejet du système tonal ${ }^{41}$, jugé trop contraignant. Concernant ce deuxième aspect, il ne s'agit évidemment par de remplacer un système harmonique par un autre, et Pâque se refusera à théoriser de façon trop précise l'atonalité, martelant au fil de ses articles que la seule définition possible est par la négative : 1'atonalité consiste simplement en l'absence de tonalité. Nous retrouvons cette absence de systématisme dans l'évolution elle-même du compositeur, et sa "versatilité stylistique ", telle qu'elle a été soulignée par Christophe Pirenne (Pirenne 2005, p. 295), explique en partie le manque de reconnaissance dont a pâti Désiré Pâque.

\section{TRANSCRIPTION}

[1] À ceux que désole l'absence de conventions dans certains départements artistiques ${ }^{42}$, je déclare qu'il faut «s'affranchir des préjugés utilitaires instinctifs ${ }^{43}$, nous mettre en garde contre la tendance de l'esprit humain à faire usage des formes de l'action pour penser $»^{I} .$. Cette citation devrait être présente à tous, à tout moment. Elle est animée de l'esprit qu'il conviendrait de généraliser si c'était possible. Créateurs et auditeurs trouveraient en elle un aliment substantiel ${ }^{44}$ en même temps qu'utile pour conduire sagement leur tendance à la critique.

[2] Des concepts musicaux tels : 1'adjonction constante et l'atonalité souffrent d'être placés sous l'égide d'une règle quelle qu'elle puisse être ; manière de considérer les aspects des choses, qui, soit dit en passant, devrait régir tout ce qui est du domaine de la pure composition (création) que l'influence du fanatisme scientifico-matérialiste n'a que trop infesté. La science implique une abstraction initiale, alors que « l'Art, lui, n'est sûrement qu'une vision plus directe et plus vraie de la réalité, obtenue des sens et de la conscience ordinaires en écartant le symbolisme de généralités utiles qui les obscurcissent d'habitude, en désintéressant leur exercice du but pratique auquel d'instinct ils tendent et s'asservissent $»^{\mathrm{II}}$.

41 Pâque a décrit les caractéristiques de son langage harmonique (Pâque 1930a, p. 124-125 ; Pâque 1930b, p. 135-140). Dans le premier article, il propose également une refonte du système de notation musicale et de la terminologie usuelle : les termes " tonique ", "dominante ", " sensible " sont maintenus mais selon une toute nouvelle acception.

42 À propos du titre choisi pour cet article par Désiré Pâque (« Réponse à quelques objections »), nous n'avons pas trouvé d'objections formulées à l'encontre du compositeur dans La Revue musicale entre 1930 et 1936 (c'est-à-dire à l'époque des articles qu'il rédige pour cette revue). Ses compositions ne font l'objet d'aucun compte rendu et Arthur Hoerée, lorsqu'il consacre une étude à « L'École belge » (Hoerée 1931), ne fait que citer un certain « Paques » en passant, sans même qualifier son esthétique. Si Philippe Gilson renvoie aux efforts menés à cette époque par André Coeuroy, Paul Gilson et Louis Lavoye afin d'attirer l'attention du public sur le compositeur (Gilson 1990, p. 5), Pâque semble bien être resté largement dans l'ombre - et cela jusqu'à aujourd'hui : en témoigne le nombre restreint d'études et d'enregistrements consacrés à sa musique.

43 Pâque écrit « insintctifs" .

I [Les notes auxquelles renvoie un chiffre romain figurent dans le texte original de Pâque] Edouard LE Roy : la Peànsée [sic] intuitive (Boivin), page 20. [Édouard Le Roy (1870-1954) est l'auteur d'Une philosophie nouvelle. Henri Bergson (1912) et de La pensée intuitive. Au-delà du discours (1929) ; partisan comme Bergson d'une pensée vivante centrée sur l'immédiat, Le Roy est aussi ami du philosophe et il le remplacera au Collège de France et à l'Académie française.]

44 Pâque écrit « subtantiel».

II Edouard LeROY [sic], op. cit., p. 51. 
[3] « L'Intelligence toujours préoccupée de savoir sous quelle ancienne rubrique elle cataloguera n'importe quel objet nouveau $»^{\mathrm{III}}$, doit vouloir dépasser ce par quoi elle se limite elle-même. Je sais bien que " l'affirmation et la répétition " ${ }^{\mathrm{IV}}$ de principes d'apparence immuable qui se retrouvent tant dans les œuvres consacrées que dans les formules théoriques toujours semblables et toujours semblablement enseignées, jointes " à l'exemple et au prestige ${ }^{\vee}$ sont des puissances contre lesquelles on ne résiste qu'au prix d'un effort de volonté opiniâtre. Sans doute. C'est pourtant cet effort qui s'impose : toujours en garde, toujours veiller. Par cette discipline constante la libération de l'esprit qui en résultera fera voir l'évanescence du convenu, de la règle, de maintes idoles.

[4] Si, prenant position sur un plan supérieur, celui où véritablement la musique règne et doit régner, sur lequel les théories n'ont pas droit de cité, où résident certaines puissances complémentaires " de l'entendement " ${ }^{\mathrm{VI}}$, il apparaîtra clairement que l'Adjonction constante telle que je l'ai présentée naguère dans la Revue Musicale ${ }^{\mathrm{VII}}$ est une illimitation de la conception musicale, plus en rapport avec la durée de l'univers, "durée ou invention, élaboration continue de l'absolument nouveau »VIII.

[5] Incomparablement moins transcendante que l'adjonction constante, l'Atonalité, simple technique nouvelle pour l'utilisation de l'échelle des sons, suscite - contrairement à l'adjonction constante - maintes réflexions plus inattendues les unes que les autres, provoque des étonnements qui se traduisent par des questions étranges, décevantes.

[6] « Lorsque l'artiste exerce librement sa fonction créatrice, n'imaginons pas qu'il s'abandonne à un jeu de fantaisie toute subjective : il réalise des virtualités latentes au sein du donné, il fait passer à l'acte des puissances réelles de perception, il contribue ainsi à

III H. Bergson, L'Évolution créatrice (Alcan), p. 53. [La citation initiale de Bergson est celle-ci : « Mais cette croyance [en la science universelle] est naturelle à l'intelligence humaine, toujours préoccupée de savoir sous quelle ancienne rubrique elle cataloguera n'importe quel objet nouveau [...]. » (Bergson [1907]1970, p. 536).]

IV $\quad \mathrm{D}^{\mathrm{r}}$ Gustave Lebon, Opinions et Croyances (Flammarion). [Dans son ouvrage Les opinions et les croyances. Genèse et évolution, Gustave Le Bon (1841-1931, davantage connu pour sa Psychologie des foules) analyse le processus de formation des croyances et des opinions au sein des groupes humains ; il définit les mécanismes d'affirmation, de répétition, d'exemple et de prestige comme essentiels à l'émergence d'une opinion, qui peut à son tour se muer en croyance.]

$\mathrm{V} \quad I d$., ib.

VI Bergson, op. cit., p. V, intr. [Bergson l'exprime en ces termes : " On dira que, même ainsi, nous ne dépassons pas notre intelligence, puisque c'est avec notre intelligence, à travers notre intelligence, que nous regardons encore les autres formes de la conscience. Et l'on aurait raison de le dire, si nous étions de pures intelligences, s'il n'était pas resté, autour de notre pensée conceptuelle et logique, une nébulosité vague [...]. Là résident certaines puissances complémentaires de l'entendement [...]. C'est dire que la théorie de la connaissance et la théorie de la vie nous paraissent inséparables l'une de l'autre. Une théorie de la vie qui ne s'accompagne pas d'une critique de la connaissance est obligée d'accepter, tels quels, les concepts que l'entendement met à sa disposition : elle ne peut qu'enfermer les faits, de gré ou de force, dans des cadres préexistants qu'elle considère comme définitifs. Elle obtient ainsi un symbolisme commode, nécessaire même peut-être à la science positive, mais non pas une vision directe de son objet » (Bergson [1907]1970, p. 492).]

VII N N 101, février 1930 [Pâque 1930a].

VIII Bergson, id., p. 11. [Bergson met ici en rapport durée et invention : " Durée signifie invention, création de formes, élaboration continue de l'absolument nouveau » (Bergson [1907]1970, p. 503).] 
nous révéler l'implicite richesse de la nature ${ }^{\mathrm{IX}}$. Cela n'a rien à voir avec «les cadres étroits et trop rigides pour ce que nous voudrions y mettre ${ }^{\mathrm{x}}$.

[7] D'aucuns réclament une théorie de l'atonalité ${ }^{45}$. Pour ceux-là ne suffit pas, sans doute, cette claire, et concise définition : l'atonalité est l'absence d'une tonalité fixe et pré-établie Je répéterai encore qu'il ne doit plus se produire " d'entraves au libre - mais ordonné développement de l'art ; par des règles arbitraires et abusives qui ne reposent ${ }^{46}$ généralement que sur la crainte du non-connu, de l'imprévu. Certes, il est des règles primordiales qu'on ne peut enfreindre. Seulement, ces règles-là sont d'un ordre supérieur, divin peut-être, ne se codifient pas, ne s'enseignent pas. Elles sont, on les sent ou on ne les sent point. Elles créent la beauté, la tendance harmonieuse des êtres et des choses, elles sont mystérieuses ainsi que leur action, ce sont elles qui, pour leurs fins élevées, se servent de l'être du génie "XII. Et encore : "règles comportant interdictions et permissions irréfragables n'ont rien et ne peuvent avoir rien de commun avec la révélation intérieure "XIII.

[8] Il est vain, pour ne pas dire plus, de vouloir, à tout prix, en matière d'art, des théories fixes et immuables. L'Art ne doit pas être confondu avec une science positive quelconque pour qui la détermination initiale exige l'indication d'un objet, d'une matière. Aussi bien, l'atonalité n'étant qu'une négation, ce qui au sens bergsonien " n'est qu'une attitude " ${ }^{\mathrm{XIV}}$, qui dit atonal exclut - par ce terme - toute théorie tonale, a fortiori je ne sais quelle théorie atonale.

[9-14] [Pâque résume la théorie de l'atonalité qu'il a formulée dans ses articles antérieurs ; il dénonce les " extravagances ", la " complexité superflue ", l'" agressivité voulue et morbide » auxquelles l'atonalité a donné naissance chez certains compositeurs.]

[15] Que l'on ne s'y méprenne pas. Les théories faites ou à faire ne sont, en conclusion, que des causes de piétinement vouées à la stérilité, à la mort. À peine écloses elles se dessèchent, tandis que s'épanouissent les nouvelles et incessantes tentatives qui affirment ainsi l'évolution créatrice.

IX Ed. Le Roy, op. cit., p. 48.

X H. Bergson, op. cit., p. [sic] [L'extrait auquel fait allusion Pâque dit en fait ceci : « En vain nous poussons le vivant dans tel ou tel de nos cadres. Tous les cadres craquent. Ils sont trop étroits, trop rigides surtout pour ce que nous voudrions y mettre (Bergson [1907]1970, p. 490). Koechlin s'était plus de dix ans auparavant référé à ce passage précis chez Bergson : « [Les innovations apportées par Schoenberg] montrent que toujours la réalité, comme écrit M. Bergson, fait craquer les cadres » (Koechlin 1922b, p. 118).]

45 On repère en effet durant l'entre-deux-guerres un effort pour définir l'atonalité (voir notamment Milhaud 1923 ou Machabey 1931, p. 35-45).

XI La Revue Musicale, N 107 (août-septembre 1930), p. 136 [Pâque 1930b, p. 135-140].

46 Pâque écrit « repose ».

XII Id., ib., p. 139.

XIII Id. N 138 (juillet-août 1933), p. 105 [Pâque 1933, p. 105-109].

XIV Bergson, op. cit., p. 311. [ Nier consiste toujours à écarter une affirmation possible. La négation n'est qu'une attitude prise par l'esprit vis-à-vis d'une affirmation éventuelle » (Bergson [1907]1970, p. 738).] 
BIBLIOGRAPHIE

a) Références citées

Antliff, Mark (1993), Inventing Bergson. Cultural Politics and the Parisian Avant-Garde, Princeton, Princeton University Press.

Antliff, Mark (2011), "Shaping Duration. Bergson and Modern Sculpture », The European Legacy, vol. $16, \mathrm{n}^{\circ}$ 7, p. 899-918.

Azouvi, François (2007), La gloire de Bergson. Essai sur le magistère philosophique, Paris, Gallimard.

Barrès, Maurice (1920), "Stendhal et la musique ", La Revue musicale, vol. 1, nº 1 (novembre), p. 2227. Disponible dans la banque de données du PHEM : http://lmhsbd.oicrm.org/media/ARTBAMd-1920-01.pdf.

Benda, Julien (1918), Belphégor. Essai sur l'esthétique de la présente société française, Paris, Émile-Paul Frères.

Bergson, Henri ([1907]1970), L'évolution créatrice, Paris, Presses Universitaires de France.

Bergson, Henri (1911), «Introduction », dans William James, Le pragmatisme, traduit de l'anglais par E. Le Brun, Paris, Flammarion.

Bergson, Henri ([1934]1970), La pensée et le mouvant, Paris, Presses Universitaires de France.

Burwick, Frederick, et Paul Douglass (1992), The Crisis in Modernism. Bergson and the Vitalist Controversy, Cambridge, Cambridge University Press.

Caron, Sylvain (2010), "L'Abbaye de Charles Koechlin, une nouvelle poétique du religieux », dans Philippe Cathé, Sylvie Douche et Michel Duchesneau (dir.), Charles Koechlin. Compositeur et humaniste, Paris, Vrin, p. 175-184.

Chennevière, Georges (1919), "La musique contemporaine et le chant choral », Le Ménestrel, vol. 81, $\mathrm{n}^{\circ} 9$ (12 décembre), p. 77-78. Disponible dans la banque de données du PHEM : http://1mhsbd. oicrm.org/media/ART-CHGc-1919-01.pdf.

Combe, Dominique (2004), «La "gloire" de Bergson », Études, vol. 10, no 401, p. 343-354, www.cairn. info/revue-etudes-2004-10-page-343.htm, consulté le 16 janvier 2019.

Douglass, Paul (1983), "The Gold Coin. Bergsonian Intuition and Modernist Aesthetics », Thought, vol. $58, \mathrm{n}^{\circ} 229$, p. 234-250.

Duchesneau, Michel (2006), "Introduction », dans in Charles Koechlin, Esthétique et langage musical, écrits présentés et annotés par M. Duchesneau, Sprimont, Mardaga, p. 9-35.

Fink, Hilary L. (2012), Bergson and Russian Modernism 1900-1930, Evenston, Northwestern University Press.

Gilson, Philippe (1990), "Désiré Pâque (1867-1939), compositeur liégeois méconnu », Bulletin de la Société liégeoise de Musicologie, vol. 70 (juillet), p. 2-18.

Hoérée, Arthur (1931), " L’École belge », La Revue musicale, vol. 12, nº 117-118 (juillet-août), p. 8996. Disponible dans la banque de données du PHEM : http://lmhsbd.oicrm.org/media/ARTHOA-1931-02.pdf.

Koechlin, Charles (1921), " D'une vaine dispute - La musique plaisir de l'esprit ou jouissance sensuelle ", La Revue musicale, vol. 2, n5 (mars), p. 219-241. Disponible dans la banque de données du PHEM : http://1mhsbd.oicrm.org/media/ART-KOC-1921-01.pdf.

Koechlin, Charles (1922a), «Le cas Berlioz », La Revue musicale, vol. 3, nº 4 (février), p. 118-134.

Koechlin, Charles (1922b), "Évolution et tradition. À propos du Pierrot lunaire de M. Schoenberg », Le Ménestrel, vol. 84, n ${ }^{\circ} 11$ (17 mars), p. 117-118. Disponible dans la banque de données du PHEM : http://lmhsbd.oicrm.org/media/ART-KOC-1922-02.pdf.

Koechlin, Charles (1923), «Paul Dupin », La Revue musicale, vol. 4, nº 3 (janvier), p. 227-241. Disponible dans la banque de données du PHEM : http://1mhsbd.oicrm.org/media/ART-KOC-1923-04.pdf. 
Koechlin, Charles (1924), «Au sujet de l'insurrection contre la sensibilité et de l'article de M. J.-R. Bloch ", Le Monde musical, octobre, p. 323-324.

Koechlin, Charles (1926), «Le temps et la musique », La Revue musicale, vol. 7, no 3 (janvier), p. 4562, désormais dans Koechlin 2006, p. 219-224. Disponible dans la banque de données du PHEM : http://lmhsbd.oicrm.org/media/ART-KOC-1926-01.pdf.

Koechlin, Charles (1927), « De l'impressionnisme (?) chez Claude Debussy », Le Monde musical, vol. 38, $\mathrm{n}^{\circ} 3$ (mars), p. 91-94. Disponible dans la banque de données du PHEM : http://lmhsbd.oicrm.org/ media/ART-KOC-1927-02.pdf.

Koechlin, Charles (1929a), «Du rôle de la sensibilité dans la musique », La Revue musicale, vol. 10, n 3 (janvier), p. 200-221. Disponible dans la banque de données du PHEM : http://lmhsbd.oicrm.org/ media/ART-KOC-1929-01.pdf.

Koechlin, Charles (1929b), "La sensibilité dans la musique contemporaine, I », La Revue musicale, vol. $10, \mathrm{n}^{\circ} 4$ (février), p. 55-63. Disponible dans la banque de données du PHEM : http://1mhsbd. oicrm.org/media/ART-KOC-1929-03.pdf.

Koechlin, Charles (1929c), "La sensibilité dans la musique contemporaine, II », La Revue musicale, vol. 10, n 5 (mars), p. 138-149.

Koechlin, Charles (1931), "Musique et mathématique », La Revue musicale, vol. 12, no 115 (mai), p. 424-439. Disponible dans la banque de données du PHEM : http://lmhsbd.oicrm.org/media/ ART-KOC-1931-01.pdf.

Koechlin, Charles (1934), «La leçon de Claude Debussy », La Revue musicale, vol. 15, no 142 (janvier), p. 1-20. Disponible dans la banque de données du PHEM : http://lmhsbd.oicrm.org/media/ARTKOC-1934-01.pdf.

Koechlin, Charles (2006), Esthétique et langage musical, écrits présentés et annotés par M. Duchesneau, Sprimont, Mardaga.

Koechlin, Charles (2009), Musique et société, écrits présentés par M. Duchesneau, Sprimont, Mardaga.

Lalo, Charles (1908), Esquisse d'une esthétique musicale scientifique, Paris, Alcan.

Lalo, Charles (1941), "Promesses et carences de l'esthétique bergsonienne », Revue de métaphysique et de morale, vol. 48, $\mathrm{n}^{\circ} 4$ (octobre), p. 301-314.

Laloy, Louis (1904), «Claude Debussy », La Revue musicale. Revue d'histoire et de critique, vol. 4, $\mathrm{n}^{\circ} 4$ (15 février), p. 106-111.

Laloy, Louis (1905a), « Les idées de M. Houdard ", Mercure musical, vol. 1, n 3 (15 juin), p. 138139.

Laloy, Louis (1905b), « Le drame musical moderne ", Mercure musical, vol. 1, no 6 (1 ${ }^{\text {er }}$ août), p. 233250.

Laloy, Louis (1910), "Claude Debussy et le debussysme », Revue musicale S.I.M., vol. 6, n 8-9 (aoûtseptembre), p. 507-519.

Laloy, Louis (1914), « M. Henri Bergson et la musique », Comcedia (19 février), p. 3.

Laloy, Louis (1929), «La musique dans l'éducation moderne », Le Courrier musical, vol. 31, n 14-15 (15 juillet- er $^{\text {eoût}}$ ), p. 459-460.

Landormy, Paul (1910), «M. Claude Debussy et l'avenir de la musique française », Le Courrier musical, vol. 13, no 3 (1 $1^{\text {er }}$ février), p. 98-101.

Landormy, Paul (1922), "Belphégor ou le règne des femmes ", Le Courrier musical, vol. 24, $\mathrm{n}^{\circ} 18$ (15 novembre), p. 333-334. Disponible dans la banque de données du PHEM : http://lmhsbd.oicrm. org/media/ART-LAPc-1922-01.pdf.

Landry, Lionel (1930), "Anticipations - Une visite à Phono-City », La Revue musicale, vol. 11, $\mathrm{n}^{\circ} 106$ (juillet), p. 33-38. Disponible dans la banque de données du PHEM : http://lmhsbd.oicrm.org/ media/ART-LAL-1930-01.pdf. 
Le Norcy, Félix (1925), « Le réalisme musical (contre-manifeste au surréalisme littéraire) », Le Courrier musical, vol. 27, $\mathrm{n}^{\circ} 8$ (15 avril), p. 215-216. Disponible dans la banque de données du PHEM : http://1mhsbd.oicrm.org/media/ART-LEFb-1925-01.pdf.

Machabey, Armand (1931), "Dissonance, polytonalité, atonalité », La Revue musicale, vol. 12, n 116 (juin), p. 35-45. Disponible dans la banque de données du PHEM : http://1mhsbd.oicrm.org/media/ ART-MAAa-1931-04.pdf.

Marcel, Gabriel (1925), "Bergsonisme et musique », La Revue musicale, vol. 6, nº 5 (mars), p. 219229. Disponible dans la banque de données du PHEM : http://lmhsbd.oicrm.org/media/ARTMAGe-1925-01.pdf.

Milhaud, Darius (1923), "Polytonalité et atonalité », La Revue musicale, vol. 4, nº 4 (février), p. 2944. Disponible dans la banque de données du PHEM : http://lmhsbd.oicrm.org/media/ARTMID-1923-02.pdf.

Milz, Manfred (2011), « Bergsonian Vitalism and the Landscape Paintings of Monet and Cézanne. Indivisible Consciousness and Endlessly Divisible Matter », The European Legacy, vol. 16, n 7 , p. 883-898.

Pâque, Désiré (1930a), " Notre esthétique ", La Revue musicale, vol. 11, no 101 (février), p. 119131. Disponible dans la banque de données du PHEM : http://lmhsbd.oicrm.org/media/ARTPADa-1930-01.pdf.

Pâque, Désiré (1930b), » L'atonalité, ou mode chromatique unique », La Revue musicale, vol. 11, nº 107 (août-septembre), p. 135-140. Disponible dans la banque de données du PHEM : http://lmhsbd. oicrm.org/media/ART-PADa-1930-02.pdf.

Pâque, Désiré (1932), «Le mystère dans l'art », La Revue musicale, vol. 13, no 126 (mai), p. 362-365.

Pâque, Désiré (1933), "Musique pure, libre et disciplinée », La Revue musicale, vol. 14, nº 138 (juilletaoût), p. 105-109. Disponible dans la banque de données du PHEM : http://lmhsbd.oicrm.org/ media/ART-PADa-1933-01.pdf.

Pâque, Désiré (1934), "La question du rythme ", La Revue musicale, vol. 15, n 147 (juin), p. 7980. Disponible dans la banque de données du PHEM : http://lmhsbd.oicrm.org/media/ARTPADa-1934-01.pdf.

Pâque, Désiré (1935), "Réponse à quelques objections ", La Revue musicale, vol. 16, n¹54 (mars), p. 238-240. Disponible dans la banque de données du PHEM : http://1mhsbd.oicrm.org/media/ ART-PADa-1935-01.pdf.

Pâque, Désiré (1936), « Musique pure », La Revue musicale, vol. 17, nº 168 (septembre-octobre), p. 293294. Disponible dans la banque de données du PHEM : http://lmhsbd.oicrm.org/media/ARTPADa-1936-01.pdf.

Pirenne, Christophe (2005), «Pâque, Marie, Joseph, Léon, Désiré », dans Nouvelle Biographie nationale, vol. 8, Bruxelles, Académie royale des sciences, des lettres et des beaux-arts de Belgique, p. 295.

Poincaré, Henri (1908a), «Comment on invente. Le travail de l'inconscient », Le Matin, 24 décembre, p. 1.

Poincaré, Henri (1908b), Science et méthode, Paris, Flammarion.

Souvtchinsky, Pierre (1932), "Sur la musique d'Igor Markévitch ", La Revue musicale, vol. 13, n 128 (juillet-août), p. 95-100. Disponible dans la banque de données du PHEM : http://1mhsbd.oicrm. org/media/ART-SOPa-1932-01.pdf.

Wahl, Jean (1943), [sans titre], dans Albert Béguin et Pierre Thévenaz (éd.), Henri Bergson. Essais et témoignages recueillis, Neuchâtel, La Baconnière.

Worms, Frédéric (2003), «L'art et le temps chez Bergson. Un problème philosophique au cœur d'un moment historique », Mil neuf cent. Revue d'histoire intellectuelle, vol. 1, nº 21, p. 153-166. 
b) Autres articles traitant du bergsonisme durant la première moitié du XX siècle dans les pays francophones (classés par ordre chronologique)

Daubresse, Mathilde (1909), "La mémoire polygonale et la musique », Bulletin français de la S.I.M., vol. 5 , n 5 (mai), p. 449-457.

Joussain, André (1911), "L'idée de l'inconscient et l'intuition de la vie ", Revue philosophique de la France et de l'Étranger, vol. 71 (mai), p. 467-493.

Joussain, André (1912), "L'expansion du bergsonisme et la psychologie musicale », Revue politique et littéraire. Revue bleue, vol. 50, n 24 (15 juin), p. 758-763.

Écorcheville, Jules (1913), «Le futurisme ou le bruit dans la musique », Revue musicale S.I.M., vol. 9 , $\mathrm{n}^{\circ} 12$ (décembre), p. 1-13.

Schloezer, Boris de (1921), "Psychologie et musique ", La Revue musicale, vol. 2, no 8 (juin), p. 244256. Disponible dans la banque de données du PHEM : http://lmhsbd.oicrm.org/media/ARTSCB-1921-01.pdf.

Koechlin, Charles (1921), "D'une nouvelle mode musicale ", La Revue musicale, vol. 2, no 10 (août), p. 132-146. Disponible dans la banque de données du PHEM : http://lmhsbd.oicrm.org/media/ ART-KOC-1921-12.pdf.

Vaillat, Léandre (1922), «Comédie des Champs Elysées », Le Ménestrel, vol. 84, nº 8 (24 février), p. 8384.

Schloezer, Boris de (1923), "Igor Stravinsky », La Revue musicale, vol. 5, n 2 (décembre), p. 97 141. Disponible dans la banque de données du PHEM : http://lmhsbd.oicrm.org/media/ARTSCB-1923-08.pdf.

Tolédano, André D. (1924), "La musique et l'intuition bergsonienne », Revue de Genève, mars, [s. p.].

Suarès, André (1924), «Musique et poésie», La Revue musicale, vol. 5, n 7 (mai), p. 3-10. Disponible dans la banque de données du PHEM : http://1mhsbd.oicrm.org/media/ART-SUA-1924-01.pdf.

Schloezer, Boris de (1924), "Réflexions sur la musique - De quelques erreurs courantes ", La Revue musicale, vol. 5, no 8 (juin), p. 269-272.

Petit, Raymond (1925), «L'identité des formes du langage sonore et de la pensée », La Revue musicale, vol. $6, \mathrm{n}^{\circ} 4$ (février), p. 152-156. Disponible dans la banque de données du PHEM : http://lmhsbd. oicrm.org/media/ART-PERa-1925-02.pdf.

Le Norcy, Félix (1926), «1925, un moment important de la vie musicale », Le Courrier musical, vol. 28, $\mathrm{n}^{\mathrm{o}} 1$ (1 $1^{\mathrm{er}}$ janvier), p. 7-8. Disponible dans la banque de données du PHEM $:$ http://1mhsbd.oicrm.org/ media/ART-LEFb-1926-01.pdf.

Landry, Lionel (1926), " La musique et ses lois ", La Revue musicale, vol. 7, no 6 (avril), p. 48-60. Disponible dans la banque de données du PHEM : http://lmhsbd.oicrm.org/media/ARTLAL-1926-01.pdf.

Jedlinski, P[aul ?] (1926), " Musique et poésie (étude critique) », Le Courrier musical, vol. 28, n 10 (15 mai), p. 269-271. Disponible dans la banque de données du PHEM : http://lmhsbd.oicrm.org/ media/ART-JEP-1926-01.pdf.

Daniel-Rops (1927), «H.-R. Lenormand et le rôle de la musique dans son théâtre », La Revue musicale, vol. $8, \mathrm{n}^{\circ} 5$ (mars), p. 225-239.

Landormy, Paul (1927), "La sensibilité musicale, d'après un livre récent », Le Ménestrel, vol. 89, n० 46 (18 novembre), p. 469-471. Disponible dans la banque de données du PHEM : http://lmhsbd.oicrm. org/media/ART-LAPc-1927-02.pdf.

Bouvier-Ajam, Maurice (1932), "Psychologie et musique ", Le Ménestrel, vol. 94, no 24 (10 juin), p. 249-250. Disponible dans la banque de données du PHEM : http://lmhsbd.oicrm.org/media/ ART-BOMa-1932-02.pdf. 
Bouvier-Ajam, Maurice (1932), «Métaphysique et musique », Le Ménestrel, vol. 94, n 48 (25 novembre), p. 473-474. Disponible dans la banque de données du PHEM : http://1mhsbd.oicrm.org/media/ ART-BOMa-1932-01.pdf.

Malherbe, Dom. B. de (1933), « Aux sources premières du rythme et de la musique », La Revue musicale, vol. $14, \mathrm{n}^{\circ} 132$ (janvier), p. 16-30. Disponible dans la banque de données du PHEM : http://1mhsbd. oicrm.org/media/ART-MABb-1933-01.pdf.

Bouvier-Ajam, Maurice (1933), "Morale et musique ", Le Ménestrel, vol. 95, no 12 (24 mars), p. 121122. Disponible dans la banque de données du PHEM : http://lmhsbd.oicrm.org/media/ARTBOMa-1933-01.pdf.

Landry, Lionel (1934), " Le commentaire musical », La Revue musicale, vol. 15, n 151 (décembre), p. 19-27. Disponible dans la banque de données du PHEM : http://lmhsbd.oicrm.org/media/ARTLAL-1934-01.pdf.

Maritain, Jacques (1936), "Sur la musique d'Arthur Lourié », La Revue musicale, vol. 17, n 165 (avril), p. 266-271. Disponible dans la banque de données du PHEM : http://lmhsbd.oicrm.org/media/ ART-MAJh-1936-01.pdf.

Marcel, Gabriel (1936), "Dukas et le transhumain », La Revue musicale, vol. 17, n 166 (mai-juin), p. 90-92. Disponible dans la banque de données du PHEM : http://lmhsbd.oicrm.org/media/ARTMAGe-1936-01.pdf. 